\title{
Erratum to: The high diversity of Lotus corniculatus endosymbionts in soils of northwest Spain
}

\author{
Marta Marcos-García ${ }^{1}$ - Esther Menéndez ${ }^{1}$ Xavier Cruz-González ${ }^{1}$. \\ Encarna Velázquez ${ }^{1,2} \cdot$ Pedro F. Mateos ${ }^{1,2} \cdot$ Raúl Rivas $^{1,2}$
}

Published online: 23 January 2016

(C) Springer Science+Business Media Dordrecht 2016

\section{Erratum to: Symbiosis}

DOI 10.1007/s13199-015-0368-5

This paper was unfortunately published with error. The geographical origin of strain MAF 303099 was Japan, not New Zealand as was written in the text and in Figures 2, 3 and 4. The corrected paragraphs as well as the updated figures are shown below.

"The strain CSLC22N representing RAPD pattern type V clustered with the type strain of M. erdmanii USDA $3471^{\mathrm{T}}$ (cluster III). This cluster also included strains isolated in Sweden (Ampomah and Huss-Danell 2011), Norway (Gossmann et al. 2012), Uruguay (Sotelo et al. 2011) and two strains nodulating $L$. corniculatus, R7A and MAFF 303099, isolated in New Zealand and Japan, respectively, and whose genome has been sequenced (Kelly et al. 2014; Kaneko et al. 2000). The strains isolated in Sweden are also misnamed as M. loti because the strain USDA $3471^{\mathrm{T}}$ was considered to be the type strain of this species before its reclassification as M. erdmanii (Martínez-Hidalgo et al. 2015)."

"The strain N3 isolated in Uruguay (Sotelo et al. 2011) formed an independent lineage related to the cluster II, which

The online version of the original article can be found at http://dx.doi.org/ 10.1007/s13199-015-0368-5

Encarna Velázquez

evp@usal.es

1 Departamento de Microbiología y Genética, Universidad de Salamanca Campus Miguel de Unamuno, Lab. 209, Edificio Departamental de Biología, 37007 Salamanca, Spain

2 Unidad Asociada de I+D Universidad de Salamanca (USAL)-CSIC (IRNASA), Salamanca, Spain also includes several type strains of Mesorhizobium species nodulating legumes other than Lotus. The cluster III contains the strain CSLC22N isolated in this study and several strains isolated from L. corniculatus nodules in Sweden (Ampomah and Huss-Danell 2011), the strain N33 in Uruguay (Sotelo et al. 2011) and the strains MAFF303099 and R7A isolated in Japan and New Zealand, respectively (Kaneko et al. 2000; Kelly et al. 2014). Moreover, this cluster includes the type strain M. erdmanii USDA $3471^{\mathrm{T}}$ isolated from L. corniculatus nodules."

"The analysis of the atpD gene showed that our strains are divided into four clusters with some differences in their distribution with respect to that found after $r e c A$ gene analysis (Figure 4). The strains from 16S rRNA gene cluster I, have different $a t p D$ gene sequences being particularly divergent in the atpD gene of the strain CSLC14N. This strain formed an independent lineage related to the type strain of $M$. caraganae CCBAU $11299^{\mathrm{T}}$ with $92.5 \%$ similarity. The remaining strains were phylogenetically related to the type strains of two species nodulating L. corniculatus, M. jarvisii ATCC $33669^{\mathrm{T}}$ and M. erdmanii USDA $3471^{\mathrm{T}}$. The phylogenetic lineage formed by the strain CSLC14N belongs to a cluster that also included two strains, S1302 and S789 isolated from L. corniculatus nodules in Uruguay (Sotelo et al. 2011). The strains CSLC01N, CSLC36N and CSLC28N were equidistant between $M$. jarvisii ATCC $33669^{\mathrm{T}}$ and $M$. erdmanii USDA $3471^{\mathrm{T}}$ (similarity higher than $98.5 \%$ in all cases) (Table 1). This makes difficult the identification of these strains as $M$. jarvisii, as suggested the recA gene analysis. The strain CSLC42N was also related to the type strain of $M$. jarvisii ATCC $33669^{\mathrm{T}}$ with $98.3 \%$ similarity, but in this case M. erdmanii USDA $3471^{\mathrm{T}}$ was less closely related, with $96.7 \%$ similarity (Table 1 ). This atpD gene cluster, also contains the strain CSLC22N from 16S rRNA gene cluster III, which present $97.8 \%$ similarity with respect to its closest 
relative $M$. jarvisii ATCC $33669^{\mathrm{T}}$ (Table 1). The L. corniculatus nodulating strains R7A and MAFF303099 isolated in New Zealand and Japan, respectively (Kaneko et al. 2000; Kelly et al. 2014) and the strain N105 isolated in
Uruguay (Sotelo et al. 2011) also belong to this cluster. However, the strains $\mathrm{R} 88 \mathrm{~b}$ and $\mathrm{CJ} 3 \mathrm{sym}$, isolated from L. corniculatus nodules in New Zealand, formed two different lineages that are phylogenetically divergent."

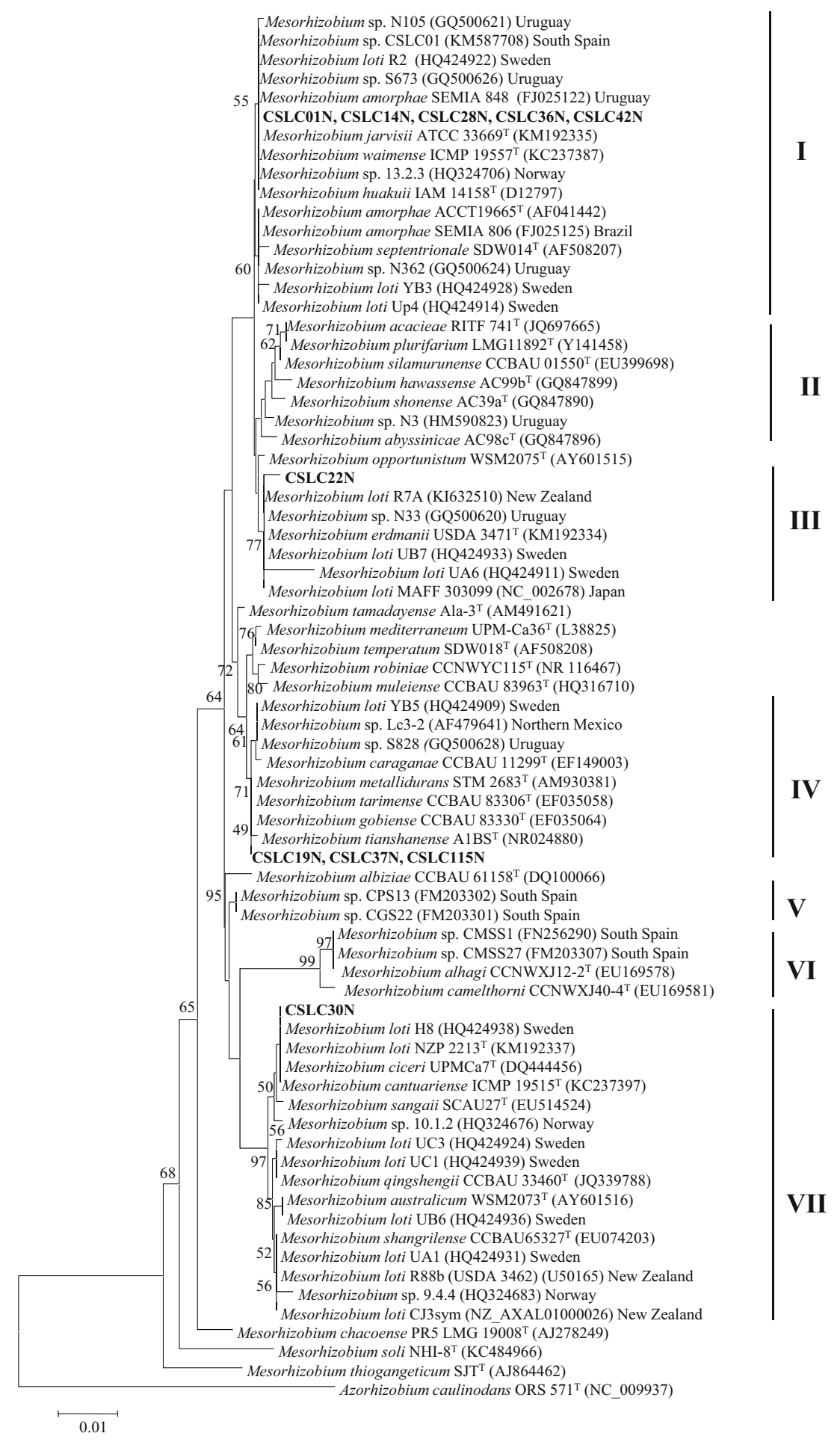


CSLC36N (KU058844)

90 Mesorhizobium jarvisii ATCC $33669^{\mathrm{T}}$ (KM192346)

68 CSLC28N (KU058842)

CSLC01N (KU058836)

CSLC42N (KU058845)

CSLC22N ( KU058841)

CSLC14N (KU058837)

51 Mesorhizobium loti MAFF303099 (BA000012) Japan 98 Mesorhizobium loti R7A (NZ_KI632510) New Zealand

Mesorhizobium australicum LMG24608 ${ }^{\mathrm{T}}$ (FR863559)

Mesorhizobium loti R88b (NZ_KI912159) New Zealand

93 Mesorhizobium loti CJ3sym A3A9DRAFT (NZ KI421462) New Zealand

Mesorhizobium sp. R-45568330 (FR772454) Belgium

Mesorhizobium huakuii CCBAU 2609 $(\mathrm{HQ} 438231)$

96 Mesorhizobium qingshengii CCBAU $33460^{\mathrm{T}}$ (JQ339757)

Mesorhizobium opportunistum WSM $2075^{\mathrm{T}}(\mathrm{CP} 002279)$

Mesorhizobium shangrilense CCBAU $65327^{\mathrm{T}}(\mathrm{EU} 672501)$

Mesorhizobium sangaii SCAU7 ${ }^{\mathrm{T}}$ (JN129442)

CSLC30N (KU058843)

Mesorhizobium ciceri USDA $3383^{\mathrm{T}}$ (AJ294367)

66 Mesorhizobium cantuariense ICMP 19515 ${ }^{\mathrm{T}}$ (KC237677)

- Mesorhizobium loti NZP 2213 ${ }^{\mathrm{T}}$ (KM192346)

Mesorhizobium robiniae CCNWYC1 $15^{\mathrm{T}}(\mathrm{GQ856501})$
Mesorhizobium mediterraneum USDA $3392^{\mathrm{T}}(\mathrm{AJ} 294369)$

- Mesorhizobium temperatum HAMBI 2583 ${ }^{\mathrm{T}}$ (DQ444305)

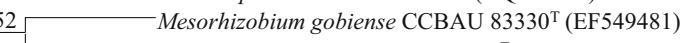

Mesorhizobium tarimense CCBAU $83306^{\mathrm{T}}$ (EF549482)

Mesorhizobium muleiense CCBAU $83963^{\mathrm{T}}$ (HQ316782)

Mesorhizobium tianshanense USDA $3592^{\mathrm{T}}$ (AJ294368)

Mesorhizobium sp. R-46108B1585 (FR772462) Belgium

Mesorhizobium sp. R-45567270 (FR772453) Belgium

Mesorhizobium caraganae CCBAU 11299 ${ }^{\mathrm{T}}$ (EU249394)

Mesorhizobium metallidurans STM2683 ${ }^{\mathrm{T}}$ (AM930382)
Mesorhizobium sp. R-457681620 (FR772450) Belgium

Mesorzhiobium amorphae ACCC 19665 ${ }^{\mathrm{T}}$ (AY688612)

CSLC115N (KU058839)

CSLC19N (KU058840)

CSLC37N (KU058838)

100|Mesorhizobium sp. R-457551590 (FR772449) Belgium

Mesorhizobium sp. R-457541588 (FR772448) Belgium

Mesorhizobium waimense ICMP $19557^{\mathrm{T}}(\mathrm{KC} 237667)$

Mesorhizobium septentrionale HAMBI $2582^{\mathrm{T}}$ (DQ444304)

Mesorhizobium acacieae RITF741 ${ }^{\mathrm{T}}$ (KM358158)

-Mesorhizobium erdmanii USDA 3471 ${ }^{\mathrm{T}}$ (AJ294371)

Mesorhizobium plurifarium USDA 4413 ${ }^{\mathrm{T}}$ (AY688611)

$74 \longrightarrow$ Mesorhizobium soli $\mathrm{NHI}-8^{\mathrm{T}}(\mathrm{KM} 188061)$

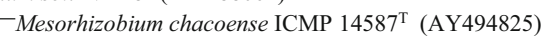

Mesorhizobium chaco

Mesorhizobium shonense AC39a ${ }^{\mathrm{T}}$ (GQ848018)

Mesorhizobium hawassense AC99b ${ }^{\mathrm{T}}$ (GQ848025)

Mesorhizobium tamadayense Ala-3 ${ }^{\mathrm{T}}$ (HE608972)

Mesorhizobium silamurunense CCBAU $01550^{\mathrm{T}}$ (EU518358)

Mesorhizobium albiziae CCBAU $61158^{\mathrm{T}}$ (EU249396)

51 Mesorhizobium sp. CPS13 (FN556460) South Spain

$100 \quad$ Mesorhizobium sp. CGS22 (FN556461) South Spain

Mesorhizobium thiogangeticum $\mathrm{SJT}^{\mathrm{T}}$ (AM04061)

TMesorhizobium camelthorni CCNWXJ40-4 ${ }^{\mathrm{T}}$ (GU220798)

99 Mesorhizobium sp. CMSS27 (FN556458) South Spain

51 Mesorhizobium sp. CMSS1 (FN556459) South Spain

Mesorzhizobium alhagi CCNWXJ12-2 ${ }^{\mathrm{T}}$ (FJ48187)

$\longmapsto 01$ 
72. CSLC36N (KU058827)

CSLC01N (KU058829)

CSLC28N (KU058835)

- Mesorhizobium jarvisii ATCC 33669 ${ }^{\mathrm{T}}$ (Km1923359) CSLC22N (KU058833)

Mesorhizobium huakuii CCBAU 2609 $(\mathrm{HQ} 438231)$

-Mesorhizobium loti R7A (AZAM01000003) New Zealand

- Mesorhizobium loti MAFF 303099 (NC_002678) Japan

$61-$ CSLC42N (KU058828)

$55^{\leftarrow}$ Mesorhizobium sp. N105 ATP (GQ500636) Uruguay

Mesorhizobium erdmanii USDA 3471 ${ }^{\mathrm{T}}$ (AJ2943931)

Mesorhizobium sp. S673 ATP (GQ500643) Uruguay

- Mesorhizobium shangrilense CCBAU 65327 $7^{\mathrm{T}}$ (EU672471)

Mesorhizobium qingshengii CCBAU 33460 ${ }^{\mathrm{T}}$ (JQ339818)

Mesorhizobium ciceri USDA $3383^{\mathrm{T}}$ (AJ294367)

- Mesorhizobium loti NZP 2313 ${ }^{\mathrm{T}}$ (KM192339)

Mesorhizobium loti R88b (JACE01000006) New Zealand CSLC30N ( KU058834)

Mesorhizobium sangaii SCAU7 ${ }^{\mathrm{T}}$ (JN129437)

Mesorhizobium australicum LMG 24608 ${ }^{\mathrm{T}}$ (JN202306)

75

Mesorhizobium loti CJ3sym (NZ AXAL01000014) New Zealand

Mesorhizobium albiziae CCBAU $61158^{\mathrm{T}}$ (DQ311090)

82 $75 \square$ Mesorhizobium sp. CPS13 (FM203309) South Spain

$22-$ Mesorhizobium sp. CGS22 (FM203310) South Spain Mesorhizobium soli $\mathrm{NHI}-8^{\mathrm{T}}$ (KM188059)

$84 \longrightarrow$ Mesorhizobium chacoense ICMP $14587^{\mathrm{T}}$ (AY493460)

\begin{tabular}{|l}
\hline CSLC14N ( KU058826) \\
Mesorhizobium sp. S948 ${ }^{\mathrm{T}}$ (GQ500646)
\end{tabular}

$94\left[\right.$ Mesorhizobium caraganae CCBAU $11299^{\mathrm{T}}(\mathrm{EU} 249379)$

$93 \_$Mesorhizobium sp. S1302 (GQ500639) Uruguay 98 Mesorhizobium sp. S789 (GQ500644) Uruguay

$59-$ Mesorhizobium sp. N362 (GQ500642) Uruguay

$86-$ Mesorhizobium septentrionale HAMBI 2582 ${ }^{\mathrm{T}}$ (DQ659498)

Mesorhizobium amorphae ACCC 19665' (AY688600)

CSLC115N (KU058831)
CSLC19N (KU058832)

$55\left[\begin{array}{c}\text { CSLC37N (KU058830) } \\ \text { Mesorhizobium metallidurans STM2683 }\end{array}\right.$

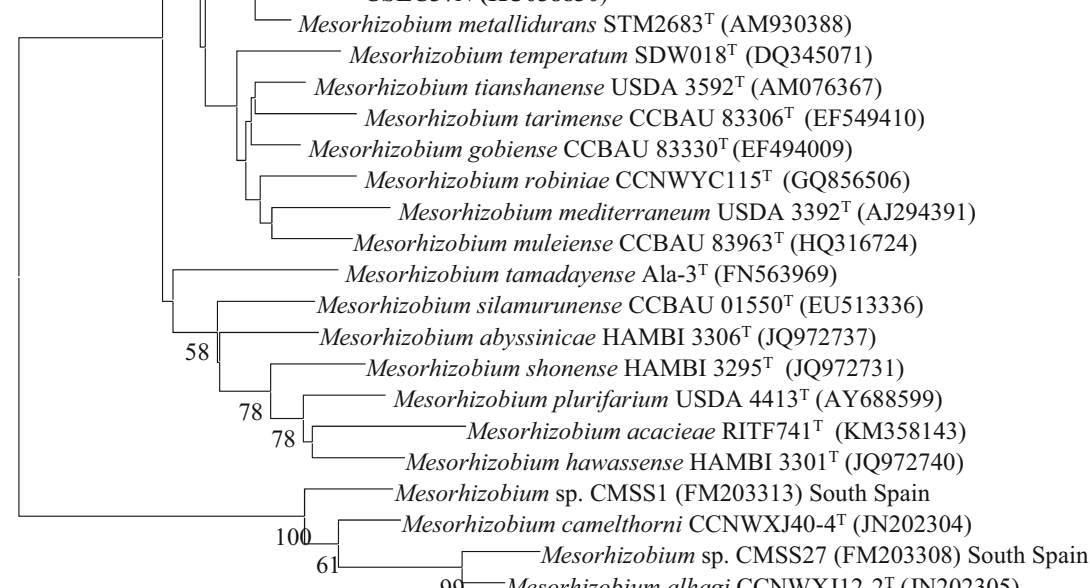

0.02 